\title{
Basic principles of design and functioning of multifunctional laser diagnostic system for non-invasive medical spectrophotometry
}

\author{
D.A. Rogatkin ${ }^{1}$, S.G. Sokolovski ${ }^{2}$, K.A. Fedorova ${ }^{2}$, N.A. Stewart ${ }^{2}$, V.V. Sidorov ${ }^{3}$, E.U. Rafailov ${ }^{2}$ \\ ${ }^{1}$ Laboratory of Medical \& Physics research, MONIKI by M.F. Vladimirskiy, Moscow 129110, RF; \\ ${ }^{2}$ Carnegie Laboratory of Physics, School of Engineering, Physics and Mathematics, University of \\ Dundee, Dundee DD1 4HN, UK; ${ }^{3}$ SPE “LAZMA" Ltd., Moscow 125252, RF
}

Keywords: multifunctional diagnostic systems; non-invasive medical spectrophotometry; laser Doppler flowmetry; optical pulse oximetry; fluorescence diagnostics; unit-module algorithm; generalised task description.

\section{Abstract}

The devising of a general engineering theory of multifunctional diagnostic systems for non-invasive medical spectrophotometry is an important and promising direction of modern biomedical engineering. We aim in this study to formalize in scientific engineering terms objectives for multifunctional laser non-invasive diagnostic system (MLNDS). The structure-functional model as well as a task-function of generalized MLNDS was formulated and developed. The key role of the system software for MLNDS general architecture at steps of ideological-technical designing has been proved. The basic principles of block-modules composition of MLNDS hardware are suggested as well.

\section{Introduction}

Last decades all over the world optical properties of biological tissues at normality and pathology (in vivo, in situ) became mainstream of intensive studies which allow us to confidently talk about the formation of a new multifunctional diagnostics as well as development of optical non-invasive diagnostic devices and systems [1-3]. In fact, it became common on medical technology market to have instruments of the optical pulse oximetry [4] and laser Doppler flowmetry [5] as well as devices for fluorescence diagnostics [6], optical coherence and diffusion tomography $[3,7]$, tissue fat detectors, blood glucose, haemoglobin, and oxyhemoglobin analysers $[1-3,8]$. Of the variety of devices of this type the most numerous group of equipment is systems implementing ideology of non-invasive medical spectrophotometry (NMS) [3], when without any bio-samples taken in accessible areas of patient body (skin, oral mucosa, blood, etc.) levels and accumulation dynamics of various biochemical markers: oxyhemoglobin, flavin respiratory enzymes, porphyrins, lipofuscin, NADH, etc. can be estimated. Moreover the most promising direction in developing such NMS devices is the creation of multifunctional (universal) laser non-invasive diagnostic systems (MLNDS) which in a single hardware implementing various methods of NMS combines fluorescence and absorption spectroscopy, laser Doppler flowmetry, etc. [9]. This allows therapist and clinicians not only receive the arithmetic sum of the diagnostic information that could be collected by each of individual method but conduct multifunctional patient examination measurements aimed for identifying subtle individual characteristics of blood flow and tissue metabolism using simultaneous and comprehensive data from different diagnostic techniques [10].

Until recently such mono- and at the best double-functional diagnostic instruments have been designing mostly empirically at lack of any serious consideration and systemic theory. It is clear that the most common theoretical basis for the creation and operation of any medical and optical-electronic devices are applicable in general and in the case of MLNDS. However it is also well known that any new class of devices especially diagnostic one has its own specificities which in varying degrees affect the entire process of developing and further exploitation starting from most common stages of the ideological and technical design and integration of the entire system. First of all it concerns to the formalised features of substantive representation of the problem, development of generalized structural and functional design, physical and mathematical model of the device. Specificity of such devices appears at formulation of

Advanced Biomedical and Clinical Diagnostic Systems IX, edited by Anita Mahadevan-Jansen, Tuan Vo-Dinh, Warren S. Grundfest, Proc. of SPIE Vol. 7890, 78901H - (c) 2011 SPIE · CCC code: 1605-7422/11/\$18 doi: $10.1117 / 12.874258$

Proc. of SPIE Vol. $789078901 \mathrm{H}-1$ 
general function of the device, as well as in architectural layout, informational, software, methodological and metrological support.

Unfortunately until recent time all these issues are poorly developed regarding to MLNDS and this article aims to at least partly fill this gap. This article uncovers main systemic and methodological principles underlying the design of MLNDS. Here we are going to give formal description of objectives for this task, structural and functional model of the generalized MLNDS and formulate its main function. According to these principles of module construction of MLNDS hardware is developed and the key role of programme-algorism maintenance of MLNDS in all matters of the overall design and synthesis of the system as a whole is established.

\section{Formal description of detailed task representation}

Following the latest trends in the development of methods and instruments for NMS [2] we consider certain MLNDS meaning by this term any particular implementation of any diagnostic device of this class regardless of inherited individual techniques of optical and laser (if using lasers as a source of radiation) NMS. According to the classical canons of Medical Instrumentation [11], any medical diagnostic device is an open medical biotechnology system (MBS), so it is a combination of biological and technical elements jointed into a uniform functional system where all elements are linked into control loop (open-loop in this case). There are two biological objects in this open-loop system: a patient and an operator-physician controlling the system analysing out-coming information from the device and deciding what medical treatments shall be prescribed to the patient.

Therefore the main goal of this study is to create multi-functional diagnostic BPS-M (MLNDS) for obtaining comprehensive information from biological object (BO) by sensing of optical and spectral signal output. Developed MLNDS contains light irradiation sources illuminating BO that can potentially create source of analogue information called initial analogue optical signal $S(x, y, \lambda, t)$, where $x$ and $y$, the spatial coordinates on the surface of the BO, $\lambda$ wavelength, $t$ - time. BOs due to their individual optical properties associated with anatomical morphological and biochemical properties of tissue, encode initial optical signal $S(x, y, \lambda, t)$ changing its basic parameters: spectral power density, shape, repetition rate and pulse duration, depth, and the function of the amplitude-frequency modulation into final optical signal $S^{*}\left(x^{*}, y^{*}, \lambda^{*}, t^{*}\right)$. Main task of MLNDS is to collect as full as possible final encoded signal $S^{*}\left(x^{*}, y^{*}\right.$, $\left.\lambda^{*}, t^{*}\right)$ filter it off external interference signals and noises and bearing information of the initial signal $S(x, y, \lambda, t)$ to calculate all the essential optical and physical BO parameters. At the final stage of data processing these optical and physical data should be converted by MLNDS into meaningful medical and biological parameters and interpreted in terms adopted in medicine [14].

Since the final optical signal coming from $\mathrm{BO}$ is also an analogue signal MLNDS should process discrete and digitise $S^{*}\left(x^{*}, y^{*}, \lambda^{*}, t^{*}\right)$ signal sampling all basic parameters: amplitude, wavelength, time intervals (the basic principle of extracting information from any analogue signals [13]). The final optical-physical and biomedical information of inspected BO should be processed in MLNDS on the base of high level of computing, processing and/or interpretive algorithms and procedures implemented in the system software [14, 15]. MLNDS operator (doctor) should be able to adopt this information in familiar form and back it up to database and for documentation.

In general this formal description of task representation is well illustrated at figure 1. That MLNDS appears to be a complex parameter-measuring BPS-M which functional characteristics and efficacy more than on two-thirds are determined by the capacity of its computing resources and user methodical software [14]. Consequently, the most important step in creating any MLNDS is the development of information, algorithmic and software support, especially at the phase of creation of general ideology of computational process in terms of diagnostic data processing and analysis.

Till now there are no uniform data processing algorithms for MLNDS. Moreover there are no even general mathematical models for calculation of the irradiation field distributed in randomly inhomogeneous medium like biological tissues which (models) are based on the algorithms that allows in any case to obtain potentially precise 
analytical solutions of task for irradiation fluxes emerging from the bio-tissue alike by parameters dense, light

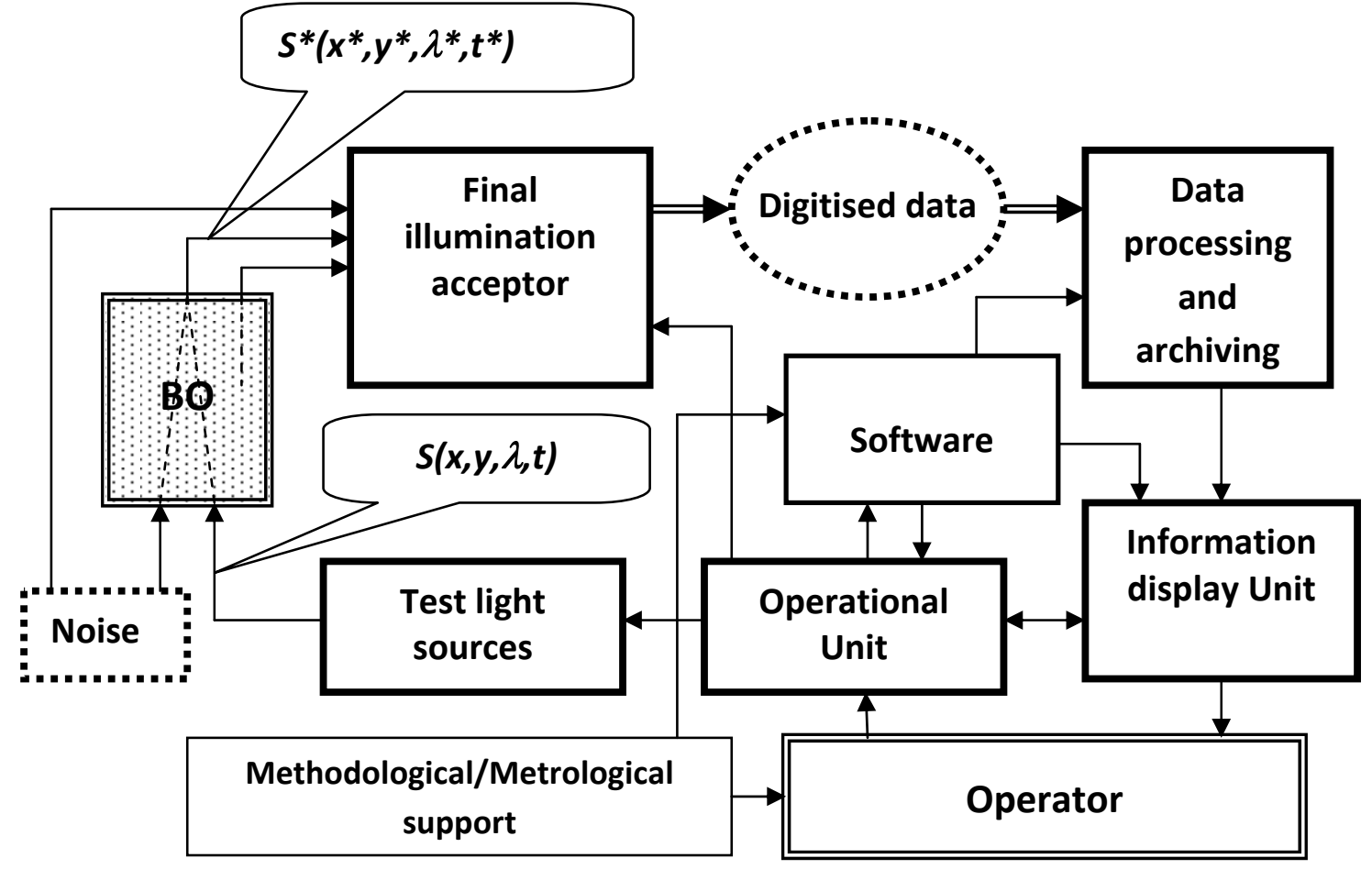

Figure 1. The formal representation of detailed task of MLNDS (explanation in text)

scattering and absorbing medium. Therefore, a large amount of theoretical research in NMS is related today to development of approximate models and algorithms that are suitable in a particular case $[1,3,16]$ including the creation of virtual physical and mathematical models of BO in terms of the theory of light propagation and scattering in turbid media [17]. Since these models are the basis of modern methods of analysis and data processing in NMS [14] their successful choice in designing phase will mainly influent on the overall clinical efficacy and diagnostic potential of MLNDS. Since all these models and algorithms could be solved so far only in particular cases of specific wavelengths, implementations of particular BO irradiation schemes and in each case defined geometry of the perception of the final optical signal $S^{*}\left(x^{*}, y^{*}, \lambda^{*}, t^{*}\right)$ then so far we can reasonably say that chosen methods and data processing algorithms "dictate" to the developer all architecture of the transmitter-receiver unit of MLNDS. The scheme displayed at figure 1 and the above arguments clearly show that system software is most important part of MLNDS where hardware is a physical base for creation and translation of optical and electronic signals being a "raw material" for the close-looped work of computing, processing and interpreting algorithms of the MLNDS software.

\section{Structural and functional model of MLNDS and its main function}

One of the first and fundamental task of design any new optic-electronic equipment including medical is the creation of adequate structural-functional model (SFM) of a new device and determining its general objective function [12, 13]. We will consider one of possible SFM (Fig.2) of generalized MLNDS based on our earlier published results [9].

In developing of SFM referring to previous section of the paper the ideology of unit-modular system with a discrete set of irradiation sources (IS) and correspondently with a discrete set of detecting wavelengths shall be used. This means that IS unit in MLNDS creates a discrete set of optical probing signals (the first level of signal discretisation by spectrum and exposure time)

$$
W_{i}=W_{i}\left(\Delta \lambda_{i}, \Delta t_{i}\right)
$$


where: $W_{i}$ - the irradiation power of $i$-th source; $\Delta \lambda_{i}$ - spectral range of $W_{i} ; \Delta t_{i}$ - time interval of $i$-th source. Irradiation formation optical scheme (IFOS) creates focused light beam with surface power density (illumination) at BO tested:

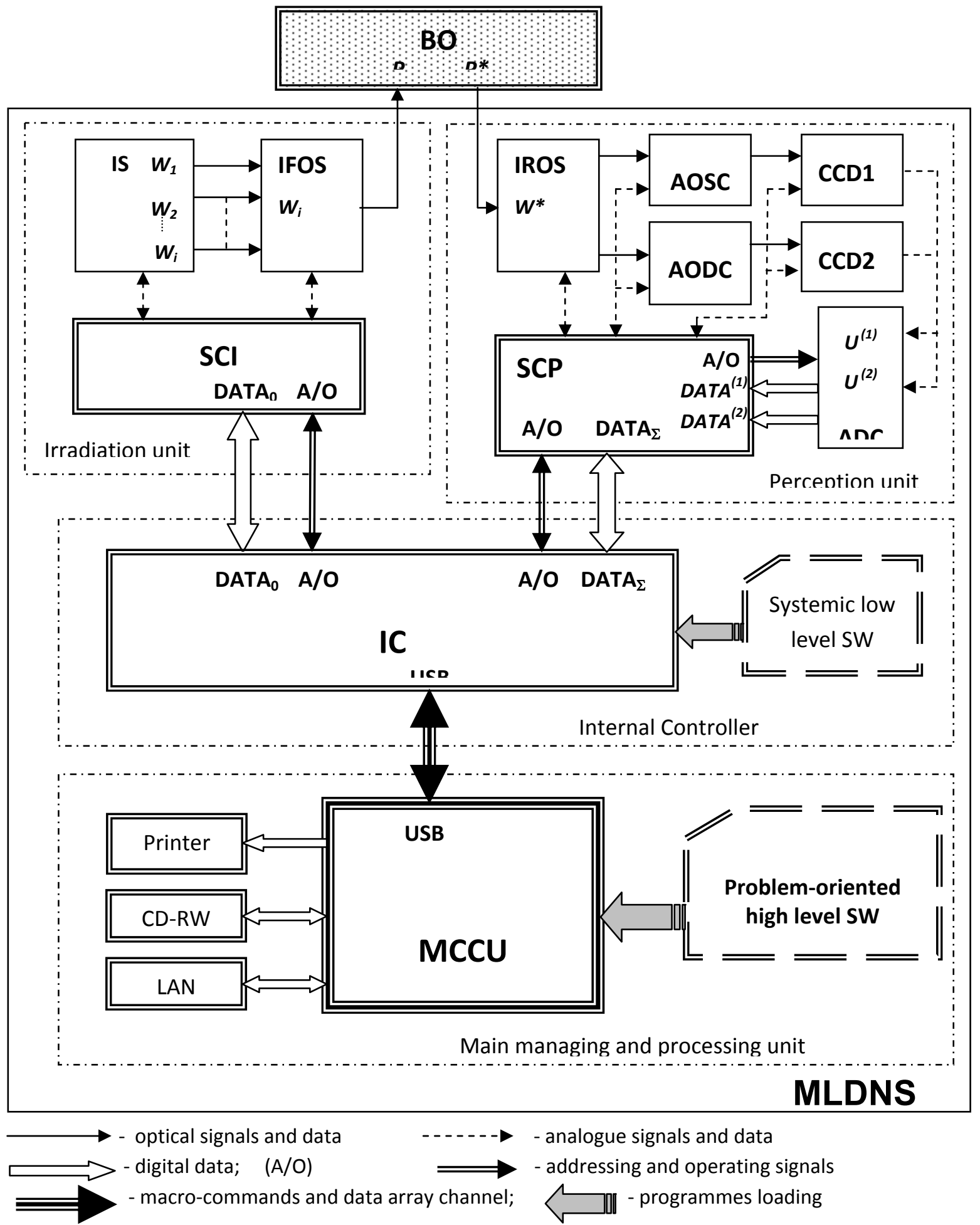

Figure 2. Structural-functional model of generalised MLNDS (refer to text for explanations). 


$$
P=f\left(x, y, \lambda, t, W_{i}\right)
$$

Analyzed BO being in fact spectral nonlinear optical filter and IS on its own (fluorescence) converts power density $P$ applied into power density $P^{*}$ of final irradiation from BO that is equivalent to coding of the initial optical signal by some encoding function:

$$
P^{*}=f^{*}\left(x^{*}, y^{*}, \lambda^{*}, t, W_{i}, W_{f 1}\right)=B\left(x \rightarrow x^{*}, y \rightarrow y^{*}, \lambda \rightarrow \lambda^{*}, t,\left\{m_{j}(t)\right\}\right) \cdot\left(P+P_{f 1}\right)
$$

where: $B$ - encoding dimensionless function of irradiation by $\mathrm{BO} ;\left\{m_{j}(t)\right\}$ - an array of biomedical, optical-physical parameters of the BO that affects the coding function; $W_{f 1}$ and $P_{f 1}$ - power and power density of the background irradiation (noise) that when passes through the BO is encoded by it.

Irradiation registration optical scheme (IROS) of MLNDS collects at some aperture angle $\omega^{*}$ final irradiation $P^{*}$ coming from BO distributed over its surface and delivers it to MLNDS. Along with this IROS collects some of the initial background irradiation $\left(W_{f 2}\right)$ not of $\mathrm{BO}$ origin. Thus, MLNDS gets total radiation power of $W^{*}$ :

$$
W^{*}\left(\lambda^{*}, t,\left\{m_{j}(t)\right\}, \ldots\right)=\int_{0}^{\omega^{*}} P^{*}\left(x^{*}, y^{*}, \lambda^{*}, t,\left\{m_{j}(t)\right\}, \ldots\right) d \omega+W_{f 2}\left(\lambda^{*}, t\right)
$$

Now general objective function of MLNDS can be formulated as determining the array $\{m j(t)\}$ of measured $W^{*}$ values as a function of signal amplitude, wavelength range and the $\mathrm{BO}$ investigation time at known function:

$$
P=f\left(x, y, \lambda, t, W_{i}\right) .
$$

In general to determine the unknown elements in $j\{m j(t)\}$ array as a function of time it is necessary to have $k \geq j$ measurements as function of time. For this purpose as shown in [9] for MLNDS perception unit would be enough to realise main functions in detecting and separating two diverse BO signals weak in amplitude but static during short time intervals (seconds) of fluorescence (fluorescent diagnostics channel) and dynamic signal of the main spectrum range $\Delta \lambda i$ (scattering and absorption spectroscopy and Doppler flowmetry channel). In the present SFM it is taken into account by introducing two analogue optical channels - static one (AOSC) and dynamic one (AODC). Discretisation of the signals by registered optical spectrum $\left(\lambda^{*}\right)$ in AOSC (second level of discretisation) is carried out by designing the channel as a polychromator with simultaneous recording of full spectrum by range of sensitive detectors like CCD. Signal discretisation in AODC is performed on the base of initial discretisation by spectrum ( $\Delta \lambda i)$ of emitting sources by choosing suitable set of lasers, and general discretisation by signal time in AOSC and AODC is performed by chosen sampling time and different sampling frequency of CCD for each channel: CCD1 and CCD2.

The amplitude signal discretisation can be carried out using a standard analogue-digital conversion (ADC) of electrical signals from CCD of perception unit and transmits data arrays for temporary storage, sorting and further processing into the memory of internal controller (IC). The same controller provides MLNDS with low-level distribution of control commands and data between perception unit and illumination unit. Low-level electronic control of units and modules perception and illumination units is covered by specialized control units (SCI and SCP). The final high-level data processing and communication of MLNDS with operator is made by the main control and computing system (MCCU) on the basis of modern high-speed computer. Thus the system signal processing $W^{*}$ in MLNDS leads to creation of two main sets of voltage readings $\{U(n)\}$ from $C C D$ and two full digital data sets $\{D A T A(n)\}$ storing information about amplitude, optical spectrum, and dynamic parameters of the signals:

$$
W^{*} \rightarrow\left\{\begin{array}{l}
\left\{U_{\Delta \lambda_{i}, \lambda^{*}}^{(1)} \rightarrow \rightarrow\left\{D A T A_{\Delta \lambda_{i}, \lambda^{*}, t_{1}}^{(1)}\right\}\right. \\
\left\{U_{\Delta \lambda_{i}, t_{2}}^{(2)}\right\} \rightarrow\left\{D A T A_{\Delta \lambda_{i}, t_{2}, t_{1}}^{(2)}\right\}
\end{array}\right\}
$$

where: $t 2<<1$ and $t 1$ is the time scales of signal discretisation at short time intervals ( $\leq$ seconds) and a relatively long time intervals (minutes, hours, days), correspondently. High-level computational algorithms tasks of MCCU are mainly 
reduced to two main functions: the restoration on the base of actual measurements of optical-physical, medical, and biological properties of $\mathrm{BO}$ :

$$
\left\{m_{j}(t)\right\}=C A\left\{\begin{array}{l}
\left\{D A T A_{\Delta \lambda_{i}, \lambda^{*}, t_{1}}^{(1)}\right\} \\
\left\{D A T A_{\Delta \lambda_{i}, t_{2}, t_{1}}^{(2)}\right\}
\end{array}\right\}
$$

where, conventionally, $C A$ is a computational algorithm and the interpretation of the computed data array $\{m j(t)\}$ in terms of biology and medicine with the probabilistic classification of the situation:

$$
\{C S\}=I A\left[\left\{m_{j}(t)\right\}\right]
$$

Where: $I A$ is interpretive algorithm and $\{C S\}$ is an informational text array of clinical situation observed for that particular survey.

Such a representation of the SFM of MLNDS allows at following stages of design to isolate reasonably and explicitly basic subtasks to be solved: computational algorithms, parametric synthesis of units and blocks of MLNDS, etc. as well as to create already at the step of the ideological and technical design virtual simulative mathematical MLNDS models.

\section{Principles of design of unit-module MLNDS}

MLNDS SFM presented tells a developer the most logical simple and optimal way of further design-structural elaboration of the MLNDS. The way to create an integrated unit-modular system that individual structural-functional elements are quite typical for many optic-electronic devices.

The opportunity for such approach was considered from analysis of recent publication containing description and technical specification of various existing optic-electronic units and their scheme-technical, technological etc. solutions. Also the general trends in global instrument development have been investigated reviewing the advertising publications (magazines "Opto \& Laser Europe", "Photonics Spectra", "Europhotonics" etc.). Finally this analysis suggests that along with a traditional manufacturers of individual components (chips, lenses, etc.) and final products (full scale equipment) a new class of the producers appears which was not evident in mid-1970-1980's, manufacturers of individual universal optic-electronic subunits and functional blocks. For example, "Point Source Ltd." and "Photonics Products" (USA) offers the finalised different wavelengths laser modules with electronic drivers, perceiving computer macro commands for direct embedding these modules into an integral automated system. "Piezosystem Jena GmbH" also produces a system for positioning and alignment of optical elements for be imbedded into integral complex systems. "Andor Technology Co." offers CCD detectors and sensors made with use of so-called open "OEM" technology for direct coupling of signals to every computer through typical PC connections. "Acton Research Corp." produces spectrophotometry modules with various sensitivity and spectral resolution from located on the board with standard "PCI" connector to be installed on PC to highly sensitive mono -and polychromator with built-in electronic alignment, $C C D$, analogue amplifiers, and ADC modules be capable to work with any PC.

Thus for the manufacturer of final medical diagnostic equipment such as MLNDS, today there is no need to develop from scratch all the basic components and units of the device. It is enough to choose the module (unit) with most suitable technical characteristics on the market and adapt it to the input and output parameters required for developing a new system. This allows to reduce the design of individual MLNDS elements to engineering of classical optic-electronic and laser elements. Using wide world experience in this area as well as databases produced to the date units and modules of optic-electronic, laser and medical devices, all of these tasks can mainly be reduced (on 85-90\%) to adaptation and interfacing of well known scheme-technical approaches into combined MLNDS.

So scientifically-based concept of MLNDS design has got basic principles of:

a) maximal standardization of individual units and modules of the MLNDS;

b) maximal functional specialization of units and modules of the MLNDS;

c) structural-functional hierarchy of the units and modules within MLNDS; 
d) optimal sharing of hardware and software functions between MLNDS units and modules

e) obedience of all these modules and systems to higher in hierarchy controller (PCs);

f) up-grating of the system with more efficient elements.

\section{Conclusion}

As it can be seen from all described above, the main peculiarity of the MLNDS design is an existence of a large number of problem-oriented computing and interpretive algorithms that at very strong extent determine the appearance and functionality of the system as a whole. The proposed structural-functional model and objective function of the generalized MLNDS consider these features and at the early stages of MLNDS design allow fully formalizing and explicitly highlighting the basic subtasks of the subsequent integration of the system, and also creating mathematical simulation model of the MLNDS. Another feature of the diagnostic systems of this class is the method of optical spectroscopy of biological tissues requiring discretisation of signals by spectrum, amplitude, and characteristic time. This imposes certain restrictions on the total number of the channels in MLNDS and their performance rate. On the other hand, MLNDS hardware itself is not very much specific regarding to the current level of development of optics, electronics and laser technology. Therefore these days the design of certain units of the MLNDS can be reduced to the classical optic-electronic and laser engineering.

Acknowledgements: This work was supported by FP7-PEOPLE-2009-IAPP project (MEDILASE, \#251531) and Russian FASI project (contract \#02.442.11.7269).

\section{References}

1. Rogatkin, D.A. "The laser clinical diagnostics as one of the perspective branch of biomedical radio-electronics and medical physics of the next millennium". Biomed. Radio-Electronics, (3), 34-41. (1998).

2. Rogatkin, D.A., Lapaeva, L.G., "Prospects for development of non-invasive spectrophotometry medical diagnostics", Biomed. Engineering, 37(4), 217-222. (2003).

3. Tuchin, V.V., [Handbook of optical biomedical diagnostics], SPIE Press, Bellingham, Washington, USA, (2002).

4. Russian State Standard ISO 9919-99, "Medical Puls-Oximeters. Technical requerements and methods of examination", Moscow, State Standard Press, (2000).

5. Krupatkin, A.I. and Sidorov, V.V., [Laser Doppler flowmetry of blood], Meditcina-Press, Moscow, (2005).

6. Loschenov, V.B., et al. "Portable spectroscopic system for fluorescent diagnostics and photodinamic therapy", Russian Chemical J, XLII(5), 50-53. (1998).

7. Gladkova, N.D., Shakhova, N.M., Sergeev A.M. [Handbook on optical coherent tomography], PhysMath Literature Press, Moscow. (2007).

8. Anderson R.R., Parrish B.B. "The optics of human skin", J. of Inv. Dermatology, 77(1), 13-19. (1981).

9. Gorenkov R.V., RogatkinD.A. et al. "A method of determination of functional condition of biological tissues and a diagnostic system to realize that." Russian patent No. 2234242. (2002).

10. Tchernyi, V.V., et al., "Complex non-invasive spectrophotometry in examination of patients with vibration disease," Photonic Therapeutics and Diagnostics II. SPIE Proc., 6078, 607828, (2006).

11. Ahutin V.M., [Biotechnical systems: theory and projecting], LGU-Press, Leningrad. (1981).

12. Ahutin V.M., Lurie O.B. et al. [Theory and projecting of electronic medical diagnostic apparatus], LGU-Press, Leningrad. (1980).

13. Mosyagin, G.M., Nemtinov, V.B., Lebedev, E.N. [General theory of optical-electronic systems] Engineering-Press. Moscow. (1990).

14. Rogatkin D.A. "Basic principles of organization of system software for multifunctional noninvasive spectrophotometric diagnostic devices and systems." Biomed. Engineering, 38(2), 61-65. (2004).

15. Bessonov A.S., Kolbas Yu. Yu., Rogatkin D.A. "Virtual diagnostic apparatus in medical noninvasive spectrophotometry." Technology of living systems, 4(1), 50-57. (2007)

16. Rogatkin, D.A. "A specific feature of the procedure for determination of optical properties of turbid biological tissues and media in calculation for non-invasive medical spectrophotometry." Biomed. Engineering, 41(20), 5965. (2007).

17. Ishimaru, A. [Wave propagation and scattering in random media], Academic Press, New-York. (1981). 